\title{
Glomus tumours - painful subungual lesion
}

\author{
AHMP Anuruddha ${ }^{1}$, BL Perera ${ }^{2}$, MB Samarawickrama ${ }^{3}$, MNA Madurawa ${ }^{4}$ \\ ${ }^{1}$ Registrar in Surgery, ${ }^{2}$ Consultant Surgeon, ${ }^{3}$ Senior Registrar in Surgery, ${ }^{4}$ Consultant \\ Histopathologist, Karapitiya Teaching Hospital, Galle.
}

\section{Introduction}

Glomus body is a controlled ateriovenous anastomosis or shunt between terminal vessels. Its main function to regulate peripheral blood flow in digits. There is a central coiled canal known as Suqnet - Hoyer canal which is lined by endothelial cells. It is surrounded by longitudinal and circular smooth muscle fibers, containing rounded epithelial like glomus cells. Glomus tumour arises from a cluster of smooth muscle cells of the glomus body with faintly easinophilic cytoplasm and pale, large nucleus. It is a hamartoma.

\section{Case report}

A 42 year old woman presented with a painful lesion in the nail bed of her right fourth finger of 8 years duration. She had been experiencing episodes of excruciating episodic pain since for the last two years. She is a teacher and had given up writing in the black board as it caused excruciating pain. She also experienced that cold water was able to reproduce the pain compressing either side of the finger reduced the intensity of pain. On examination the nail was slightly reddish purple and there was extreme tenderness over it. There were high Doppler signals over the lesion suggestive of a vascular lesion. Pain was reduced by compression of the digital arteries.

All surgery transungual approach revealed shiny encapsulated swelling of $2 \mathrm{~mm}$ in diameter. Histopathological examination confirmed it as a benign glomus tumour (Fig 1, \& 2).

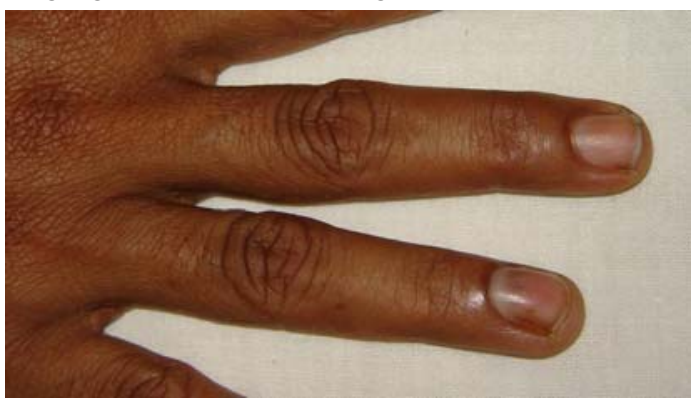

Figure 1

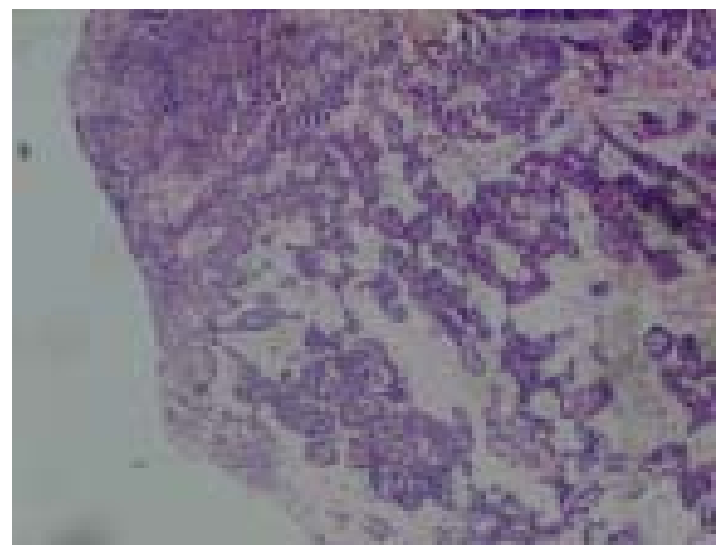

Figure 2

\section{Discussion}

Glomus tumours present with a long duration of symptoms before treatment averaging 2-3 years. They have a classical triad of clinical features paroxysmal pain, tenderness, and cold sensitivity $[1,2]$. Hildreth's sign, Love's test and cold sensitivity are the commonly used clinical tests to diagnose Glomus tumours.

Hildreth's sign is the disappearance of pain after application of a tourniquet proximally on the arm and found to have $92 \%$ sensitivity and 91\% specificity [3]. Eliciting pain with a pointer in precise area is known as Love's test and has $100 \%$ sensitivity. Cold test is, keeping the affected site in ice water for one minute which reproduce the characteristic pain.

Except MRI, other laboratory and imaging techniques will not provide any diagnostic features [4].

Glomus tumours are commonly located in the subungal region of fingers. However glomus tumours in wrist fore arm, foot, tip of the spine and glans penis is not uncommon. There are reported cases of glomus tumours in the gastrointestinal tract, trachea, and vagina. 
Females are commonly affected and usually have a solitary lesion. Twenty five percent of cases are multiple and common in men. These multiple glomus tumours are known as glomangiomas. Very few reported cases are available with atypical and malignant changes. Tumour with a deep location and size of more than $2 \mathrm{~cm}$ or atypical mitotic figures or moderate to high nuclear grade and five mitotic figures or more per high power field are the criteria for diagnosis of malignant changes [5].

Only treatment option for Glomus tumour is surgical excision but with multiple tumours it should be confined to symptomatic lesions. Complete excision is more difficult as there are micro infiltrations in to adjacent normal tissue which can lead to recurrence unless adequate margin is excised [6].

Periungual approach for tumours in the peripheral region and transungal approach followed by careful repair of the nail bed for tumours in the central region is indicated to minimise nail deformity.

\section{Reference}

1. Schuuraman AH, Bos KE. Glomus tumours of digits. Netherlands Journal of Surgery 1987; 39: 132-3.

2. Corrado EM, Passarti U, Meessore L, Lanza F. Thermographic diagnosis of glomus tumours. Journal of Hand Surgery 1982; 14: 21-4.

3. Giele H. Hildreth's test is a reliable clinical sign for diagnosis of Glomus tumours. Journal of Hand Surgery 2002; 27: 157-8.

4. Drap JL, Idy Peretti I. Standard and high resolution MRI of glomus tumour of toes and finger tips. Journal of American Academic Dermatology 1996; 35: 550-5.

5. Atypical and malignant glomus tumours analysis of 52 cases with a proposal for the reclassification of glomus tumours, American Journal of Surgical Pathology 2001; 25: $1-12$

6. Takata H, Ikuta Y, Shod O, Kimori K. Treatment of subungual glomus tumours. Journal of Hand Surgery 2001; 6: 25-7. 